\title{
REFERENCES
}

1. A. A. Albert, Quasigroups. I, Trans. Amer. Math. Soc. vol. 54 (1943) pp. 507519.

2. - Quasigroups. II, Trans. Amer. Math. Soc. vol. 55 (1944) pp. 401-419.

3. O. Ore, Chains in partially ordered sets, Bull. Amer. Math. Soc. vol. 49 (1943) pp. 558-566.

United States Naval Academy, Postgraduate School

\section{TWO ELEMENT GENERATION OF A SEPARABLE ALGEBRA}

\section{A. A. ALBERT}

The minimum rank of an algebra $A$ over a field $F$ is defined to be the least number $r=r(A)$ of elements $x_{1}, \cdots, x_{r}$ such that $A$ is the set of all polynomials in $x_{1}, \cdots, x_{r}$ with coefficients in $F$. In what follows we shall assume that $A$ is an associative algebra of finite order over an infinite field $F$.

It is well known that $r(A)=1$ if $A$ is a separable field over $F$ and that $r(A)=2$ if $A$ is a total matric ${ }^{1}$ algebra over $F$. Over fourteen years ago I obtained but did not publish the result that $r(A)=2$ if $A$ is a central division algebra over $F$. The purpose of this note is to provide a brief proof of the generalization which states that if $A$ is any separable algebra over $F$ then $r(A)=1$ or 2 according as $A$ is or is not commutative.

We observe first that a commutative separable ${ }^{2}$ algebra $Z$ is a direct sum of separable fields and that there exists a scalar extension $K$ over $F$ such that $Z_{K}$ has a basis $e_{1}, \cdots, e_{n}$ over $F$ for pairwise orthogonal idempotents $e_{i}$. If $u_{1}, \cdots, u_{n}$ is a basis of $Z$ over $F$ and $x=a_{1} u_{1}+\cdots+a_{n} u_{n}$ the powers $x^{i}$ have the form

where the determinant

$$
x^{i}=\sum_{j=1}^{n} b_{i j} u_{j} \quad(i=1, \cdots, n),
$$

$$
d\left(a_{1}, \cdots, a_{n}\right)=\left|b_{i j}\right|
$$

is a polynomial in the parameters $a_{1}, \cdots, a_{n}$. If $c_{1}, \cdots, c_{n}$ are any

Received by the editors April 13, 1944.

1 See page 95 of my Modern higher algebra.

2 The definition of a separable algebra given below reduces to a direct sum of fields in the commutative case. When $F$ is nonmodular the concept of semisimple algebra and separable algebra coincide. 
distinct elements of $K$ the quantity $x_{0}=c_{1} e_{1}+\cdots+c_{n} e_{n}$ is known ${ }^{1}$ to generate the diagonal algebra $Z_{K}$, that is $Z_{K}=F\left[x_{0}\right]$ has a basis $x_{0}, x_{0}^{2}, \cdots, x_{0}^{n}$. If we express the quantities $e_{1}, \cdots, e_{n}$ linearly in terms of $u_{1}, \cdots, u_{n}$ we see that $x_{0}$ is a value of $x$ for values $a_{i 0}$ of the $a_{i}$ in $K$. The linear independence of $x_{0}, x_{0}^{2}, \cdots, x_{0}^{n}$ implies that $d\left(a_{10}, \cdots, a_{n 0}\right) \neq 0$. Then $d\left(a_{1}, \cdots, a_{n}\right)$ is not identically zero and thus there exists a quantity $x$ in $Z$ such that $Z$ has a basis $x, x^{2}, \cdots, x^{n}$ over $F, Z=F(x), r(Z)=1$.

An algebra $A$ is called a separable ${ }^{2}$ algebra if $A$ is a direct sum of simple components $A_{k}$ such that the center of every $A_{k}$ is a separable field over $F$. If $x$ and $y$ are in $A$ we define

$$
F[x, y]
$$

to mean the set of all polynomials

$$
\sum_{i=1, \cdots, m}^{j=1, \cdots, q} a_{i j} x^{i} y^{j}
$$

Only a finite number of the power products $x^{i} y^{j}$ are linearly independent and each $F[x, y]$ is a linear subspace of $A, m$ and $q$ may be be selected so that $F[x, y]$ has order $m q$ over $F$.

A separable algebra has a unity quantity $e$ and if $A=F[x]$ then $A$ has a basis $x^{0}=e, x, \cdots, x^{n-1}$ over $F, A=F[x, e]$. Also $A$ is commutative. If $A$ is not commutative and $A=F[x, y]$ then $e=x[f(x, y)] y$ and thus $x$ and $y$ must be nonsingular. Note then that $A$ has a basis of power products $x^{i} y^{j}$ where $i=0, \cdots, m-1$ and $j=0, \cdots, q-1$. We use these results in the proof of our principal

THEOREM. If $A$ is a separable algebra which is not commutative then $r(A)=2, A=F[x, y]$ for nonsingular elements $x$ and $y$ such that $F[x]$ is separable.

We first study the case where $A$ is the direct product of a total matric algebra $M$ of degree $g$ and a division algebra $D$ of degrees $s$ over a separable center $C$ over $F$. It is well known ${ }^{3}$ that $D$ contains a maximal separable subfield $W=C\left[x_{0}\right]$ of degree $s$ over $C$ and that $W=F\left[x_{0}\right]$. The algebra $Q=\left(e_{11}, \cdots, e_{g g}\right)$, whose basis consists of a set of primitive idempotents of $M$ whose sum is its unity element $e$, has the property that $Z \neq Q \times W$ is separable and commutative, and so $Z=F[x]$. If $K$ is a scalar splitting field over $C$ of $D$ the algebra $Z_{K}$ contains $n=g s$ primitive pairwise orthogonal idempotents whose sum

${ }^{8}$ See Theorem 4.18 of my Structure of algebras, Amer. Math. Soc. Colloquium Publications, vol. 24, New York, 1939. 
is the unity element $e$ of the total matric algebra $A_{K}$ of degree $n$ over $K$. Also $Z=C[x], Z_{K}=K[x]$ and it is known ${ }^{1}$ that there exists a quantity $y_{0}$ in $A_{K}$ such that

$$
A_{K}=K\left[x, y_{0}\right],
$$

that is, the power products $x^{i} y_{0}^{j}$ taken for $i, j=1, \cdots, n$ are linearly independent in $K$. If $p=n^{2}$ and $u_{1}, \cdots, u_{p}$ are a basis of $A$ over $C$ we may write $y=a_{1} u_{1}+\cdots+a_{p} u_{p}$ and express the powers $x^{i} y^{j}$ in the form

$$
\begin{aligned}
z_{k}=x^{i} y^{j} & =\sum_{h=1} b_{k h} u_{h} \\
& (k=i+j n-n ; i, j=1, \cdots, n),
\end{aligned}
$$

for $b_{k h}$ in $F$. The determinant $d\left(a_{1}, \cdots, a_{p}\right)=\left|b_{k h}\right|$ is a polynomial in $a_{1}, \cdots, a_{p}$ with coefficients in $C$ which is not identically zero since it is not zero for values $a_{10}, \cdots, a_{j 0}$ which define $y_{0}$. It follows that $A=C[x, y]$. But $C[x]=F[x]$ so that $A=F[x, y]$.

We finally consider a separable algebra $A$ which is the direct sum of simple components $A_{1}, \cdots, A_{t}$. By the proofs above every component $A_{k}=F\left[x_{k}, y_{k}\right]$, where $y_{k}$ is the unity quantity $e_{k}$ of $A_{k}$ when $A_{k}$ is commutative, $Z_{k}=F\left[x_{k}\right]$ is separable. The algebra $Z$ which is the direct sum of $Z_{1}, \cdots, Z_{t}$ is a commutative separable algebra and so $Z=F[x]$. Let $y=y_{1}+\cdots+y_{t}$. Since $F[x]$ contains every $x_{k}$ the linear space $F[x, y]$ contains $x_{\mathbf{k}}^{\mathbf{k}} y^{j}=x_{\mathbf{k}}^{\mathbf{k}} y_{\mathbf{k}}^{j}$. For $x_{k}^{j}=x_{\mathbf{k}}^{j} e_{k}$ and $e_{k} y^{j}$ $=\left(e_{k} y\right)^{j}=y_{k}^{j}$. It follows that $F[x, y]$ contains every $A_{k}$ and that $F[x, y]=A$.

The University of Chicago 